\title{
A double-crystal scheme with full compensation of the Migdall effect
}

\author{
Dmitry Frolovtsev* and Sergey Magnitskiy \\ Lomonosov MSU, Faculty of physics and ILC, 119991 Moscow, Russia
}

\begin{abstract}
The double-crystal scheme for polarization-entangled photon pairs generation is presented. The negative impact of the Migdall effect on the polarization entanglement is fully compensated in the scheme. The theory for quantifying of entanglement decrease as the action of the Migdall effect is formulated.
\end{abstract}

Sources of polarization-entangled photon pairs are key stone in experimental quantum optics [1] and applications [2]. Spontaneous parametric down-conversion [3,4] (SPDC) based sources are the most successful class of sources for implementation in quantum technologies. They are bright [5], highly-entangled [6] and provide opportunities for engineering [7]. The double-crystal scheme [8] shares all of these advantages simultaneously. There are some decoherence mechanisms $[9,10]$ negatively affecting the produced entanglement, but only the Migdall effect [11] remains uncompensated. In this paper we present a method for full compensation of the Migdall effect in the double-crystal scheme.

We analyze the generalized double crystal scheme (Fig. 1). In the scheme the two nonlinear crystals may be arbitrarily rotated around the pump wavevector direction. We show that there are special angles of the crystal rotation [12] where the Migdall effect does not affect the entanglement of generated photon pairs.

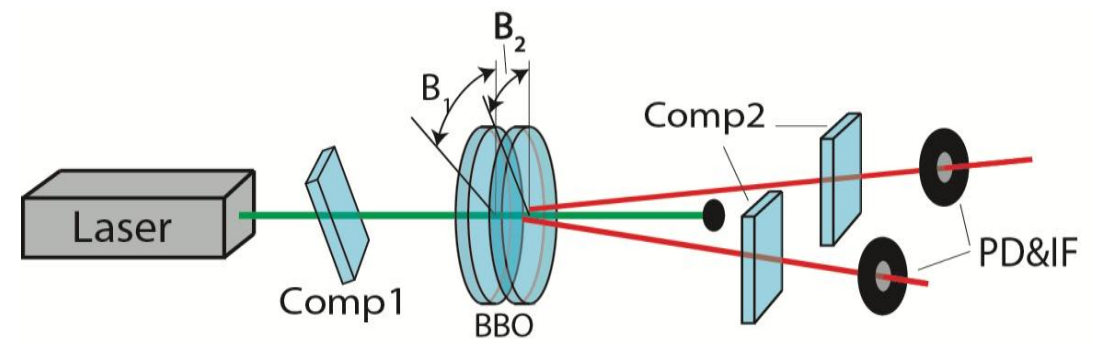

Fig. 1. The double-crystal scheme. Comp1 and Comp2 are birefringent compensation plates, BBO nonlinear crystals, PD\&IF - diaphragms and interference filters, $\mathrm{B}_{1}$ and $\mathrm{B}_{2}-$ angle of rotation of the nonlinear crystals around the pump wavevector direction

We theoretically show that the polarization quantum state of generated photon pairs has the form

\footnotetext{
*Corresponding author: dfrolovtsev@ gmail.com
} 


$$
p=\frac{1}{2}\left(\begin{array}{ccccc}
\sin ^{2} \delta \sin ^{2} \epsilon & 0 & 0 & \sin \delta \sin \epsilon \\
0 & & \cos ^{2} \delta \sin ^{2} \epsilon & 0 & 0 \\
& 0 & 0 & \sin ^{2} \delta \cos ^{2} \epsilon & 0 \\
\sin \delta \text { sin } \epsilon & 0 & 0 & 1+\cos ^{2} \delta \cos ^{2} \epsilon
\end{array}\right)
$$

where $\delta$ and $\varepsilon$ are the angles between the polarization of photons borned in the first and the second nonlinear crystals in the signal and idler spatial modes, respectively. Parameters $\delta$ and $\varepsilon$ are to be calculated according to the formulas for the Migdall effect [11]. The dependence of Tangle on the $B_{1}$ and $B_{2}$ angles is shown on Fig. 2. The calculation was made for the BBO crystals pumped at the wavelength of $532 \mathrm{~nm}$, crystallographic angle $\Theta=29.6^{\circ}$, external SPDC half-opening angle is $6.9^{\circ}$.

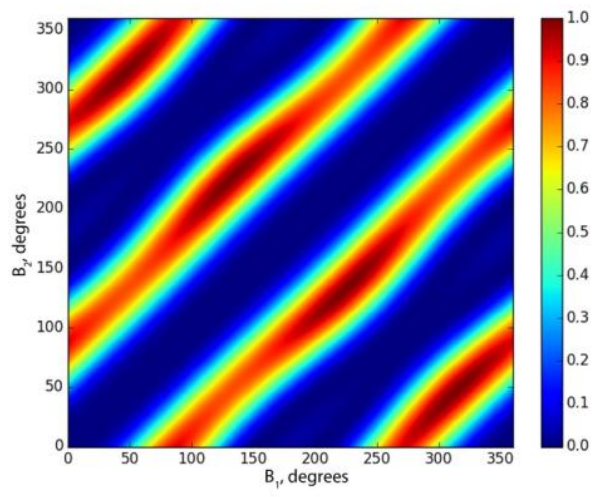

Fig. 2. Dependence of Tangle on $B_{1}$ and $B_{2}$

Fig. 2 shows there are four crystal positions where the Tangle has its maximum value of 1 . In these positions the angles of rotation satisfy $B_{1}=-B_{2}$ up to $360^{\circ}$ rotations. We show [12] that in the optimal positions the polarization generated in the first crystal is orthogonal to the polarization generated in the second crystal in the signal as well as in the idler mode (i.e. $\delta=\epsilon=90^{\circ}$ ). As a result, such source acts as if there is no Migdall effect. The only action of the Migdal effect is the basis rotation, and it does not affect the degree of entanglement. Thus, the negative action of the Migdall effect on the degree of polarization entanglement in the generalized double-crystal scheme can be fully compensated.

This work was supported by the Foundation for the Advancement of Theoretical Physics and Mathematics "BASIS" (grant \#18-2-6-189-1) and RFBR (grant \#18-02-00849)

\section{References}

1. Scully, M. O., Zubairy, M. S., Quantum optics, New York, Cambridge U.P., 1997

2. O'brien, J. L., Furusawa A., Vučković J., Nature Photonics 3.12, 687 (2009)

3. D.N. Klyshko // Talk at the All-Union Workshop on Nonlinear Properies of Media (Chernogolovka, Moscow region, 1966)

4. D.N. Klyshko, LETP Lett. 6.23 (1967)

5. M. Hentschel, H. Hübel, A. Poppe et al., Opt. Expr. 17.25, 23153-23159 (2009)

6. A. Fedrizzi, T. Herbst, A. Poppe, et al., Opt. Expr. 15.23, 15377-15386 (2007)

7. S. Carrasco, A. Sergienko, B. Saleh, et al, Phys. Rev. A 73.6, 063802 (2006)

8. P. Kwiat, E. Waks, A. White, et al., Phys. Rev. A 60.2, R773 (1999)

9. J. Altepeter, E. Jeffrey and P. Kwiat, Opt. Expr. 13.22, 8951-8959 (2005)

10. R. Rangarajan, M. Goggin and P. Kwiat, Opt. Expr. 17.21, 18920-18933 (2009)

11. A. Migdall, JOSA B 14.5, 1093 (1997)

12. D. Frolovtsev, S. Magnitskiy, patent RU \#2636808 C1 (2016) 